\title{
Prevalencia y factores de riesgo de incontinencia urinaria de esfuerzo en mujeres deportistas nulíparas.
}

\author{
Camila María Marín Mora ${ }^{1}$ y Mariela Fonseca Chaves ${ }^{1}$ \\ camyma124@hotmail.com \\ marielafonsecach@hotmail.com \\ ${ }^{1}$ Universidad de Costa Rica \\ Envío original: 2018-10-09 Reenviado: 2019-05-22 Aceptado: 2019-08-28 \\ Publicado: 2019-10-08 \\ Doi: https://doi.org/10.15517/pensarmov.v17i2.35033
}

\begin{abstract}
Resumen
Objetivo: Descubrir la prevalencia de la Incontinencia Urinaria de Esfuerzo (IUE) en las mujeres nulíparas deportistas y qué factores de riesgo se asocian a la misma. Metodología: Se hizo una búsqueda minuciosa en bases de datos utilizando palabras claves en español, inglés y portugués. Se incluyeron solamente artículos con esta población, publicados del 2000 a 2015, y con metodologías de cohortes, casos y controles, de prevalencia, revisiones bibliográficas, y ensayos clínicos aleatorios. La muestra de estudios comprendió 20 artículos, la mayoría en el idioma inglés. Resultados: La prevalencia de IUE encontrada fue muy variada ya que se presentaron casos en los que solo el $9 \%$ de mujeres evaluadas la padecían, mientras que, en otros, la totalidad de la población la presentaba. No obstante, las investigaciones con una prevalencia alta concordaron en que las poblaciones estudiadas practicaban deportes de alto impacto y eran de países nórdicos. En cuanto a los factores de riesgo (FR) personales, se destacaron el estreñimiento, la lumbalgia, la inhabilidad de detener el flujo urinario y el asma considerados también factores predictores. Por su parte, los deportes con mayor predisposición a padecer IUE fueron los considerados de alto impacto, así como aquellos que involucraban atajar un objeto. Otros factores que mostraron resultados importantes fueron: la cantidad de años de practicar el deporte, el nivel y el volumen semanal de entrenamiento. Conclusión: A pesar de que aún no existe suficiente evidencia científica que explique la relación de la IUE en mujeres nulíparas deportistas, esta investigación demuestra que el deporte juega un papel importante en el desencadenamiento de este padecimiento.
\end{abstract}

Palabras clave: prevalencia, factor de riesgo, incontinencia urinaria, deporte, mujer, nulípara.

\begin{abstract}
Objective: To discover the prevalence of the Stress Urinary Incontinence (SUI) in nulliparous female athletes and the risk factors associated. Methods: Only were included articles containing: the described population, published from 2000 to 2015, and with methodologies such as cohort studies, case-control studies, of prevalence, systematic reviews and randomized trials. The sample included 20 studies, mainly in English. Results: The prevalence of SUI varies between $9 \%$ and the total amount of participants evaluated. Despite of this, the investigations that showed the highest prevalence of SUI were found in sports involving high impact activities and were originally from the Nordics. Regarding the
\end{abstract}


personal risk factors, the constipation, the low back pain, the inability to interrupt the urine flow, and asthma were also considered predictive factors. On the other hand, the sports that involved high impact and catching objects showed a higher risk of SUI. Other factors that should be considered are: year of practice, the level and weekly volume of training. Conclusion: There is still not enough scientific evidence that explains the relationship between the SUI and nulliparous female athletes, however, this research proves that sports have an important role with the presence of SUI.

Key words: prevalence, risk factor, urinary incontinence, sport, woman, nulliparous.

\section{Resumo}

Descubrir la prevalencia de la Incontinencia Urinaria de Esfuerzo (IUE) en las mujeres nulíparas deportistas y qué factores de riesgo se asocian a la misma

Objetivo: Descovrir a prevalência da Incontinência Urinária por Estresse (IUE) nas mulheres esportivas nulíparas, e os fatores de risco relacionados. Metodologia: Foram analizados somente artigos com essa poblação, pulicados entre 2000 e 2015, e com metodologías de coortes, casos e controles, de prevalência, revisões bibliográficas, e ensaios clínicos aleatórios. Foram selecionados 20 artigos, a maioria em inglês. Resultados: A prevalência de IUE foi variada já que se apresentaron casos em que só um $9 \%$ de mulheres examinadas tinham o padecimento, mas em outros casos a totalidade da população a padecíam. No entanto, as investigações com uma maior prevalência foram apresentadas nos exercícios intensos e nos países Nórdicos. Em relação aos fatores de risco pessoais, mostrou-se que os principais foram: constipação, a dor lombar, incapacidade de parar o fluxo urinário, e o asma, que também são considerados fatores preditores. A literatura apuntou que os esportes com a maior predisposição de ter IUE são os exercícios intensos e que precisem de pegar um objeto. Entretanto, a cantidade de anos de praticar o esporte, o nível e o volume semanal de treinamento, também mostraram ser fatores importantes. Conclusão: Apesar de que ainda não existe suficiente evidência científica que explique a relação entre a Incontinência Urinária por Estresse e as mulheres esportivas nulíparas, a investigação mostra que os esportes tem um papel importante na aparição deste padecimento.

Palavras-chave: Prevalência, fator de risco, Incontinência Urinária, esporte, mulher, nulipara.

Con el aumento de la participación de las mujeres a nivel deportivo profesional y no profesional, no se han tomado en cuenta las implicaciones que éste tiene en la biomecánica del cuerpo femenino, así como otros factores influyentes en el desempeño de esta (Napal et al. 2004). Estudios han señalado una lesión relacionada con el piso pélvico (PP); la incontinencia urinaria de esfuerzo (IUE), la cual corresponde a la pérdida involuntaria de orina durante acciones como toser, estornudar, en el ejercicio físico, levantamiento de peso o incluso al realizar cambios repentinos de posición (Martínez, 2012).

Algunas investigaciones en estudiantes universitarias de Educación Física en los Estados Unidos, muestran que de cada siete mujeres al menos seis presentan IUE (Nygaard, Thompson, Svengalis y Albright, 1994). Esto relacionado con otra investigación en el mismo tipo de población en Francia, que obtuvo como resultado un $62,8 \%$ de alumnas con una edad media de 21 años con presencia de IU al realizar actividades deportivas 
(Elleuch et al., 1998). Del mismo modo, en Nueva Zelanda se presentó un aumento de casos de IUE en mujeres que practican deportes de alto impacto por un tiempo prolongado.

Rial y Rieira (2012), agregan que la prevalencia de la IUE en mujeres sedentarias en comparación con mujeres deportistas resulta significativamente menor, de $2.85 \%$ frente a $31.42 \%$ respectivamente. Recientemente se confirman estos resultados en el estudio realizado en atletas brasileñas por Almeida et al. (2015), donde se compara la prevalencia de disfunciones del PP en 67 atletas principiantes contra 97 sedentarias. Las deportistas presentaron mayor prevalencia de IUE hasta en un 95\% en comparación con el grupo control.

Cabe destacar que además de la relación entre las características del deporte y la IUE, existen factores personales y asociados al estilo de vida de la persona. Entre ellos se encuentran: el tabaquismo, la dieta y el tipo de bebidas consumidas, los medicamentos utilizados y las actividades de la vida diaria como toser, estornudar, defecar, saltar y gritar (Cabrera, 2006).

Por último, la importancia de lo anteriormente descrito recae en que la IUE puede perjudicar la vida laboral, social y sobre todo deportiva de la mujer. Según Cabrera (2006), las mujeres evitan consultar al médico por dicha molestia ya que en su mayoría lo consideran normal o se sienten avergonzadas sobre su condición, sin importar el impacto que éste tiene en sus actividades de la vida diaria.

Partiendo de esto, se plantea la siguiente interrogante: ¿Cuál es la prevalencia de la IUE en las mujeres nulíparas deportistas y qué factores de riesgo se asocian a la misma?

\section{METODOLOGÍA}

Diseño. El presente estudio es una revisión bibliográfica. La recopilación de los artículos científicos se hizo en el período que transcurre entre agosto y setiembre de 2016 , mientras que el espacio estuvo definido por las bases de datos ScienceDirect, Ovid, Proquest, Cochrane Library, Springer, EBSCO Host, PEdro, Redalyc, Scielo, ICI Web Science, Scopus y PubMed.

Población. Se analizaron artículos encontrados en las bases de datos mencionadas, publicados entre los años 2000 y 2015. Además, debían estar escritos en los idiomas español, inglés y portugués, haber sido publicados en revistas científicas, y cuya población incluyera deportistas nulíparas con el padecimiento de incontinencia urinaria. Se utilizó una estrategia de búsqueda, combinando los siguientes descriptores en los tres idiomas mencionados. En español: Incontinencia urinaria de esfuerzo, femenino, factores de riesgo, prevalencia, ejercicio, y los mismos en su traducción correspondiente al inglés (stress urinary incontinence, female, risk factor, prevalence, exercise) y portugués (incontinência urinaria de esforço, mulher, fatores de risco, prevalência, exercício). La estrategia de búsqueda utilizada fue la siguiente:

- ((Incontinencia urinaria de esfuerzo) AND (ejercicio) AND (femenino) NOT (terapéutica))

- ((Incontinencia urinaria de esfuerzo) AND (ejercicio) AND (mujeres) NOT (terapéutica))

- ((Incontinencia urinaria de esfuerzo) AND (ejercicio) AND (factores de riesgo) NOT (terapéutica)) 
- ((Incontinencia urinaria de esfuerzo) AND (ejercicio) AND (prevalencia) NOT (terapéutica))

De esta manera, la población final comprendió un total de 20 artículos científicos, en su mayoría en el idioma inglés.

Criterios de inclusión. Se eliminaron todos aquellos artículos duplicados con el gestor de referencias bibliográficas Zotero. A partir de ahí, se descartan aquellos artículos que no cumplan con los criterios de inclusión descritos en la Tabla 1. Este procedimiento se realiza en 3 fases: títulos, resúmenes ejecutivos y texto completo. En el caso de los resúmenes ejecutivos, se utilizó el software "Abstrackr" para identificar palabras de mayor relevancia.

Tabla 1. Criterios de inclusión en los estudios por analizar

Estudios de cohortes, casos controles, de prevalencia, revisiones bibliográficas,
ensayos clínicos aleatorios.

Con un intervalo de confianza de al menos $95 \%$.

Publicados a partir del año 2000 hasta el 2015.

La población deben ser mujeres deportistas.

Las mujeres deben ser nulíparas y no pueden estar en periodo de gestación.

Fuente: Elaboración propia, 2016.

Validez. Se utilizaron tres diferentes metodologías para revisar la validez de los estudios: clasificarlos en nivel I, II o III según criterios relacionados con el tipo de metodología, el factor de impacto de la revista en la que fue publicado el artículo, y la zona de Bradford delimitada según la ley con el mismo nombre (Tabla 2). El artículo debía cumplir 2 de 3 criterios para clasificarse en el nivel de validez correspondiente. La segunda forma se basó en los criterios según la US Agency Healthcare Research and Quality.

Tabla 2. Criterios de validez de evidencia de los estudios recolectados.

1. Estudios aleatorizados, doble ciego, multicéntricos y meta análisis.

I 2. Factor de impacto de la revista de 1 er o 2ndo cuartil.

3. Pertenecen a la primera zona de Bradford.

1. Estudios controlados o unicéntricos.

II

2. Factor de impacto de la revista de 3er cuartil. 
3. Pertenecen a la segunda zona de Bradford.

1. Reporte de casos o editorial.

III

2. Factor de impacto de la revista de 4 to cuartil

3. Pertenecen a la tercera zona de Bradford.

Fuente: Elaboración propia, 2016.

Debido a que estas fueron insuficientes, se realizó una tercera basada en el diseño metodológico de la investigación, cuyos factores tomados en cuenta se encuentran en la Tabla 3.

Procedimiento. En cada una de las bases de datos se realizó la búsqueda utilizando la estrategia previamente descrita, adaptada a cada caso. Asimismo, se utilizó el gestor de referencias bibliográficas Zotero para la gestión automatizada de las citas bibliográficas, así como para eliminar los estudios duplicados entre las diversas bases de datos.

A partir de los títulos y resúmenes identificados, se realizó una primera selección de los potenciales artículos y, por medio de la aplicación de los criterios de inclusión, se extrajeron aquellos no necesarios. Como se mencionó anteriormente, este procedimiento se realizó en tres etapas: títulos, resúmenes y texto completo. Para aquellos artículos que no cumplieron los criterios, se explicó el motivo de su exclusión, con el propósito de disminuir y justificar el número de estudios no apropiados.

Por último, el proceso de búsqueda y selección de los artículos se sintetiza en un diagrama de flujos (Figura 1), identificando los artículos recolectados en cada fase, así como la cantidad de descartados.

Es importante destacar que tanto la revisión de los artículos como la extracción de los datos se realizó por parte de ambas investigadoras para asegurar la fiabilidad del proceso. En casos en los que hubo discrepancias, se revisó el artículo en conjunto para determinar su inclusión o exclusión. No fue necesaria la intervención de un tercer investigador en ninguna de las decisiones.

\section{RESULTADOS}

Proceso de selección de artículos. La estrategia de búsqueda arrojó 5943 artículos científicos como total de todas las bases de datos utilizadas. De ellos, 3840 eran duplicados dando un total de 2103.

Seguidamente, se procedió a revisar por medio del programa Microsoft Office Excel 2010, los títulos de los mismos justificando uno a uno la razón de descarte en los casos necesarios. En la etapa de revisión de títulos se eliminan 1898 para un total de 205 seleccionados, la mayoría porque hacían referencia al tratamiento. Posteriormente se revisan los resúmenes ejecutivos por medio del software Abstrackr, con lo cual se conservaron 80 artículos, y la razón de exclusión predominante fue la población de estudio. 
Finalmente, las investigadoras leen los 80 artículos resultantes, no obstante, más de la mitad de ellos fueron excluidos en el apartado metodológico. Además, cabe destacar que no se tuvo acceso al texto completo de dos de los artículos, por lo que se tuvieron que excluir. En total, se analizaron 20 artículos científicos cuya población incluye a deportistas nulíparas con incontinencia urinaria, que constituyen la muestra para la elaboración de esta investigación.

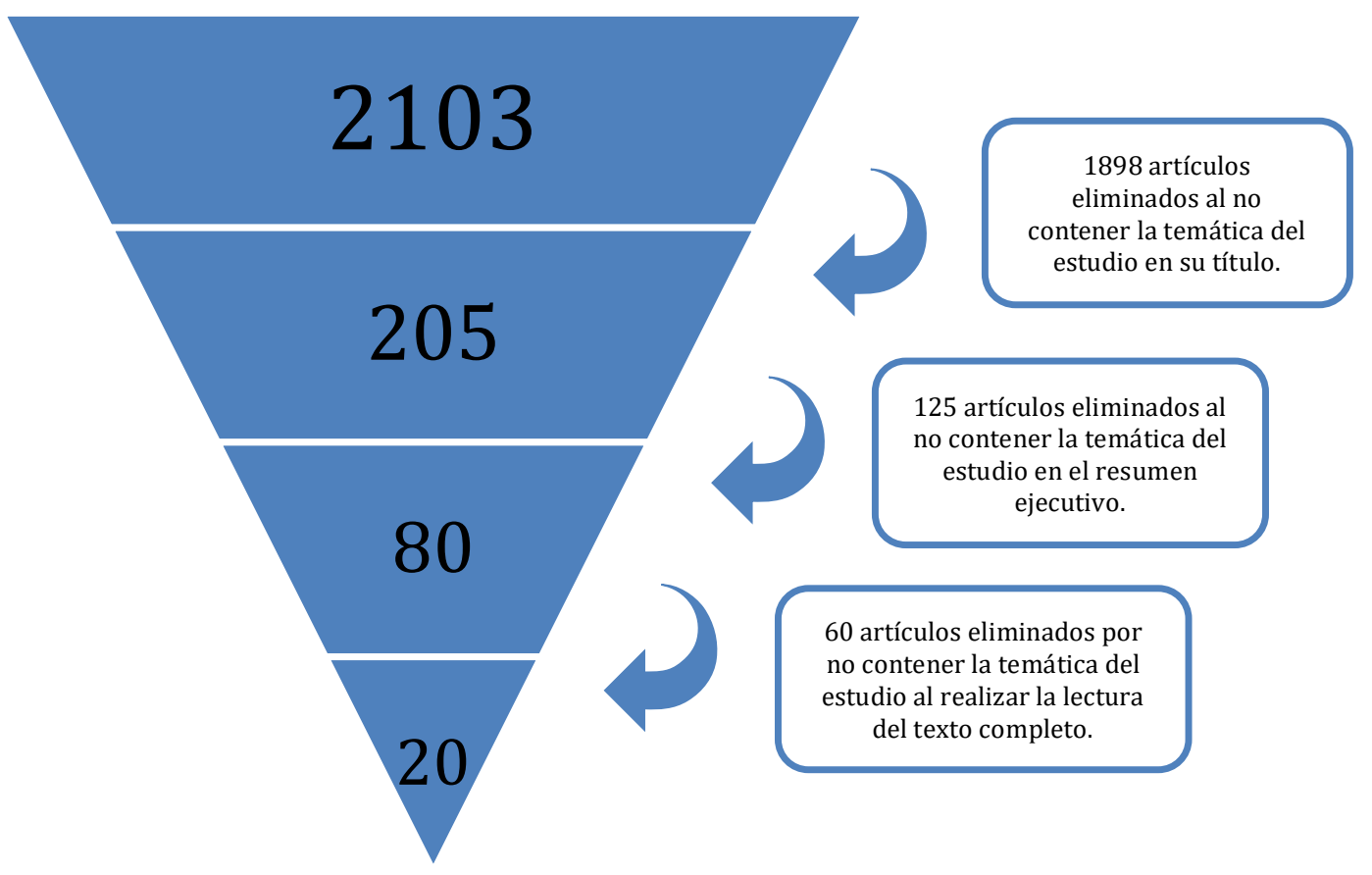

Figura 1. Proceso de selección de artículos de la búsqueda en las diferentes bases de datos. Fuente: elaboración propia

Caracterización de los artículos. La totalidad de artículos analizados fueron publicados en el idioma inglés, a excepción de uno en el idioma portugués, y publicados con mayor frecuencia en el 2007, 2008 y 2015. Además, provenían mayoritariamente de Estados Unidos, Brasil y Portugal, de tipo observacional en su mayoría.

Al analizar la validez de los estudios por medio de los aspectos metodológicos, se concluyó que cuatro de los artículos (Da Roza, Brandão, Mascarenhas, Jorge, y Duarte, 2015a, Fozzatti et al., 2012, Eliasson, Edner, y Mattsson, 2008, Dockter, Kolstad, Martin, y Schiwal 2007) tienen mayor validez debido a sus poblaciones mayores a 100, muestreo probabilístico y el control de los sesgos. Además, cabe destacar que todos los estudios mostraron ser nivel III de evidencia científica según la "US Agency for Healthcare Research and Quality". 
Tabla 3. Características metodológicas de los artículos que mostraron mayor validez en la investigación.

\begin{tabular}{|c|c|c|c|c|c|c|}
\hline Autores & Año & Población & Muestreo & $\begin{array}{c}\text { Sesgos } \\
\text { controlados }\end{array}$ & Ética & $\begin{array}{l}\text { Instrumentos } \\
\text { utilizados }\end{array}$ \\
\hline \multirow{2}{*}{$\begin{array}{l}\text { Da Roza, } \\
\text { Brandão, } \\
\text { Mascaren } \\
\text { has, } \\
\text { Jorge, y } \\
\text { Duarte, } \\
(\underline{2015 a)}\end{array}$} & \multirow[t]{2}{*}{2015} & \multirow[t]{2}{*}{386} & \multirow[t]{2}{*}{$\begin{array}{c}\mathrm{P}, \\
\text { Aleatorio. }\end{array}$} & $\begin{array}{l}\text { Sesgo de } \\
\text { medición. }\end{array}$ & \multirow{2}{*}{$\begin{array}{c}\text { Se aplica } \\
\text { consentimient } \\
\text { o informado a } \\
\text { menores. } \\
\text { Aprobado por } \\
\text { comité de } \\
\text { ética }\end{array}$} & \multirow[t]{2}{*}{$\begin{array}{l}\text { ICIQ-UI-SF, } \\
\text { Cuestionario }\end{array}$} \\
\hline & & & & $\begin{array}{l}\text { Sesgo de } \\
\text { confusión. }\end{array}$ & & \\
\hline \multirow[t]{2}{*}{$\begin{array}{l}\text { Dockter et } \\
\text { al (ㅁ007) }\end{array}$} & \multirow[t]{2}{*}{2007} & \multirow[t]{2}{*}{177} & \multirow[t]{2}{*}{$\begin{array}{c}\mathrm{P}, \\
\text { Aleatorio }\end{array}$} & \multirow[t]{2}{*}{$\begin{array}{l}\text { Sesgo de } \\
\text { medición. }\end{array}$} & \multirow{2}{*}{$\begin{array}{c}\text { Se aplica } \\
\text { consentimient } \\
\text { o informado. } \\
\text { Aprobado por } \\
\text { comité de } \\
\text { ética }\end{array}$} & $\begin{array}{l}\text { Cuestionario } \\
\text { validado }\end{array}$ \\
\hline & & & & & & $\begin{array}{l}\text { Consentimiento } \\
\text { informado }\end{array}$ \\
\hline \multirow{2}{*}{$\begin{array}{l}\text { Eliasson, } \\
\text { Edner, y } \\
\text { Mattsson, } \\
(\underline{2008)})\end{array}$} & \multirow[t]{2}{*}{2008} & \multirow[t]{2}{*}{305} & \multirow{2}{*}{$\begin{array}{c}P, \\
\text { totalidad } \\
\text { de } \\
\text { población. }\end{array}$} & $\begin{array}{l}\text { Sesgo de } \\
\text { medición. }\end{array}$ & \multirow[t]{2}{*}{$\begin{array}{l}\text { Aprobado por } \\
\text { comité de } \\
\text { ética }\end{array}$} & \multirow[t]{2}{*}{$\begin{array}{l}\text { Cuestionario } \\
\text { validado }\end{array}$} \\
\hline & & & & $\begin{array}{l}\text { Sesgo de } \\
\text { selección. }\end{array}$ & & \\
\hline \multirow{3}{*}{$\begin{array}{l}\text { Fozzatti } \\
\text { et al., } \\
(\underline{2012)}\end{array}$} & \multirow[t]{3}{*}{2012} & \multirow[t]{3}{*}{488} & $\begin{array}{c}\mathrm{P}, \\
\text { Aleatorio. }\end{array}$ & $\begin{array}{l}\text { Sesgo de } \\
\text { medición. }\end{array}$ & \multirow[t]{3}{*}{$\begin{array}{l}\text { Aprobado por } \\
\text { comité de } \\
\text { ética }\end{array}$} & $\begin{array}{l}\text { Cuestionario } \\
\text { personal }\end{array}$ \\
\hline & & & & $\begin{array}{l}\text { Sesgo de } \\
\text { selección. }\end{array}$ & & $\begin{array}{l}\text { Cuestionario de } \\
\text { actividad física }\end{array}$ \\
\hline & & & & & & $\mathrm{ICIQ}$ \\
\hline
\end{tabular}

Fuente: Elaboración propia, 2016.

Prevalencia de IUE en mujeres deportistas nulíparas.

Es importante destacar que, de la totalidad de artículos sólo se pudieron analizar 17, ya que 3 de ellos solamente incluían datos sobre factores de riesgo y no sobre prevalencia.

De estos 17 artículos, siete de ellos son investigaciones con el objetivo de evaluar la prevalencia de IUE, la cual varía entre 8 y $80 \%$ según los artículos recolectados por las investigadoras.

Con los 10 artículos restantes se analizó la población evaluada y la prevalencia absoluta y relativa obtenida en cada estudio. Los resultados se ubicaron entre los rangos de $5,7 \%$ hasta $100 \%$. Asimismo, en la mayoría de los casos, la prevalencia de IUE es mayor al $15 \%$ en sus resultados. Sumado a esto, se realiza un análisis de los artículos que presentan mayor prevalencia (> 50\%), para descubrir que el deporte que presenta más casos es el trampolinismo, y los países con mayor incidencia son los nórdicos, donde destaca Suecia con 3 investigaciones.

Factores de riesgo asociados con padecer IUE en mujeres deportistas nulíparas. A continuación, se presentan los factores de riesgo (FR) estudiados en el periodo del 2000 al 
2015 , sintetizados en un cuadro resumen y divididos en según sean características propias de la persona (Tabla 4) o si se asocian al deporte que ésta práctica (Tabla 5). En los casos en los que el estudio concluye que un elemento aumenta la probabilidad de padecer IUE en mujeres deportistas se representa con un signo de suma (+), mientras que en el caso contrario se coloca un signo de resta (-). Por otro lado, las casillas en blanco indican que los autores no evaluaron dicho factor. 
Tabla 4. Resumen de FR personales encontrados en los artículos científicos.

\begin{tabular}{|c|c|c|c|c|c|c|c|c|c|c|c|c|}
\hline Artículo & $\mathrm{n}$ & $\begin{array}{l}\text { Características } \\
\text { de la población }\end{array}$ & Edad & Raza & IMC & $\begin{array}{l}\text { Características } \\
\text { ciclo menstrual }\end{array}$ & $\begin{array}{c}\text { Morf. } \\
\text { lumbar y } \\
\text { lumbalgia }\end{array}$ & $\begin{array}{l}\text { Morf. del piso } \\
\text { pélvico (PP) }\end{array}$ & $\begin{array}{c}\text { Incapacidad } \\
\text { de detener el } \\
\text { flujo urinario } \\
\text { luego de } \\
\text { haber } \\
\text { iniciado } \\
\end{array}$ & $\begin{array}{l}\text { Hipermovilidad } \\
\text { articular }\end{array}$ & Estreñimiento & Asma \\
\hline $\begin{array}{l}\text { Capson, } \\
\text { Nashed } \\
y \\
\text { Mclean, } \\
(\underline{2011})\end{array}$ & 86 & $\begin{array}{l}\text { Deportistas de } \\
22 \text { a } 41 \text { años } \\
\text { sometidas a } \\
\text { maniobras de } \\
\text { hiperpresión } \\
\text { intraabdominal }\end{array}$ & & & & & + & & & & & \\
\hline $\begin{array}{c}\text { Da } \\
\text { Roza, } \\
\text { Branda, } \\
\text { Mascare } \\
\text { nhas, } \\
\text { Jorge y } \\
\text { Duarte, } \\
(\underline{2015 b)}\end{array}$ & 22 & $\begin{array}{l}\text { Trampolinistas } \\
\text { profesionales }\end{array}$ & & & & + & & & & & & \\
\hline $\begin{array}{c}\text { Da Roza } \\
\text { et al., } \\
(\underline{2015 c)}\end{array}$ & 12 & Futbolistas & - & & - & - & & - & & & & \\
\hline $\begin{array}{c}\text { Da Roza } \\
\text { et al., } \\
(\underline{2015 a)}\end{array}$ & 386 & $\begin{array}{c}\text { Practica de } \\
\text { deporte } \\
\text { organizado, de } \\
14 \text { a } 33 \text { años }\end{array}$ & - & & - & - & & & + & & - & \\
\hline $\begin{array}{c}\text { Da Silva } \\
\text { Borin, } \\
\text { Nunes, } \\
\text { de } \\
\text { Oliveira, } \\
(\underline{2013})\end{array}$ & 40 & $\begin{array}{c}\text { Volleyball, } \\
\text { balonmano, } \\
\text { basketball y } \\
\text { sedentarias entre } \\
\text { los } 18 \text { y } 30 \text { años. }\end{array}$ & & & & & & + & & & & \\
\hline $\begin{array}{l}\text { Eliasso, } \\
\text { Elfving, } \\
\text { Nordgrn, }\end{array}$ & 193 & $\begin{array}{l}\text { Deportistas con } \\
\text { lumbalgia entre } \\
\text { los } 17 \text { y } 45 \text { años }\end{array}$ & & & & & + & & + & & & + \\
\hline
\end{tabular}




\begin{tabular}{|c|c|c|c|c|c|c|c|c|c|c|}
\hline $\begin{array}{l}\text { Mattsso } \\
\mathrm{n},(\underline{\underline{2008}})\end{array}$ & & & & & & & & & & \\
\hline $\begin{array}{l}\text { Eliasson } \\
\text {, Edner } \\
\text { et al } \\
(\underline{2008})\end{array}$ & 305 & $\begin{array}{l}\text { Trampolinistas } \\
\text { entre } 18 \text { y } 44 \\
\text { años }\end{array}$ & & & + & & + & & + & + \\
\hline $\begin{array}{l}\text { Eliasson } \\
\text { Larsson, } \\
\text { Mattsso } \\
\mathrm{n},(\underline{2002})\end{array}$ & 35 & $\begin{array}{l}\text { Trampolinistas } \\
\text { entre } 12 \text { y } 22 \\
\text { años }\end{array}$ & + & & - & & + & & & \\
\hline $\begin{array}{l}\text { Fozzatti } \\
\text { et al., } \\
(\underline{2012})\end{array}$ & 488 & $\begin{array}{l}\text { Ejercicio de alto } \\
\text { impacto y asisten } \\
\text { al gimnasio. }\end{array}$ & & - & - & & & & & \\
\hline $\begin{array}{l}\text { Kruger, } \\
\text { Dietz, y } \\
\text { Murphy, } \\
(\underline{2007})\end{array}$ & 46 & $\begin{array}{l}\text { Deporte de alto } \\
\text { impacto y alta } \\
\text { intensidadentre } \\
19 \text { y } 39 \text { años. }\end{array}$ & & & & - & & - & & \\
\hline
\end{tabular}


Es importante destacar que tanto la raza como el IMC y la hipermobilidad articular no mostraron resultados significativos en ninguno de los artículos recolectados. Los otros factores estudiados tuvieron diversidad de conclusiones, los cuales se comentan en el apartado de discusión.

Tabla 5. Resumen de FR asociados al deporte encontrados en los artículos científicos.

\begin{tabular}{|c|c|c|c|c|c|c|c|}
\hline Artículo & $\mathrm{n}$ & $\begin{array}{l}\text { Características de } \\
\text { la población }\end{array}$ & $\begin{array}{l}\text { IU durante } \\
\text { la práctica } \\
\text { deportiva }\end{array}$ & $\begin{array}{l}\text { Tipo de } \\
\text { deporte } \\
\text { evaluado }\end{array}$ & $\begin{array}{c}\text { Características } \\
\text { del } \\
\text { entrenamiento }\end{array}$ & $\begin{array}{c}\text { Nivel de } \\
\text { entrena } \\
\text { miento }\end{array}$ & $\begin{array}{l}\text { Periodo del } \\
\text { entrenamiento }\end{array}$ \\
\hline Carls, (2007) & 86 & $\begin{array}{l}\text { Estudiantes atletas } \\
\text { de } 14 \text { a } 21 \text { años } \\
\text { practicantes de } \\
\text { deportes de alto } \\
\text { impacto. }\end{array}$ & + & & & & \\
\hline 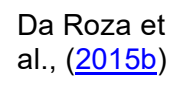 & 22 & $\begin{array}{l}\text { Trampolinistas } \\
\text { profesionales }\end{array}$ & + & & + & + & \\
\hline $\begin{array}{l}\text { Da Roza et } \\
\text { al., }(\underline{2015 c})\end{array}$ & 12 & Futbolistas & + & & & & \\
\hline $\begin{array}{l}\text { Da Roza et } \\
\text { al., (ㅁ15a) }\end{array}$ & 386 & $\begin{array}{l}\text { Practica de deporte } \\
\text { organizado, de } 14 \text { a } \\
33 \text { años }\end{array}$ & & + & + & + & \\
\hline $\begin{array}{c}\text { Da Silva } \\
\text { Borin et al., } \\
(\underline{2013})\end{array}$ & 40 & $\begin{array}{c}\text { Volleyball, } \\
\text { balonmano, } \\
\text { basketball y } \\
\text { sedentarias entre } \\
\text { los } 18 \text { y } 30 \text { años. }\end{array}$ & & + & + & & \\
\hline $\begin{array}{l}\text { Dockter et } \\
\text { al., (2007) }\end{array}$ & 177 & $\begin{array}{c}\text { Atletas univesitarias } \\
\text { de las disciplinas } \\
\text { atletismo, } \\
\text { basketball, sofbol, } \\
\text { fútbol, volleyball, } \\
\text { porrismo y } \\
\text { sedentarias. }\end{array}$ & & - & & & \\
\hline $\begin{array}{l}\text { Eliasson, } \\
\text { Edner et al } \\
\quad(\underline{2008})\end{array}$ & 305 & $\begin{array}{l}\text { Trampolinistas } \\
\text { entre } 18 \text { y } 44 \text { años }\end{array}$ & + & + & & & \\
\hline $\begin{array}{l}\text { Eliasson et } \\
\text { al., (2002) }\end{array}$ & 35 & $\begin{array}{l}\text { Trampolinistas } \\
\text { entre } 12 \text { y } 22 \text { años }\end{array}$ & + & & + & & + \\
\hline $\begin{array}{l}\text { Fozzatti et } \\
\text { al., (2012) }\end{array}$ & 488 & $\begin{array}{c}\text { Ejercicio de alto } \\
\text { impacto y asisten al } \\
\text { gimnasio. }\end{array}$ & & + & & & \\
\hline $\begin{array}{l}\text { Kruger et al., } \\
\qquad \underline{(2007)}\end{array}$ & 46 & $\begin{array}{c}\text { Deporte de alto } \\
\text { impacto y alta } \\
\text { intensidadentre } 19 \\
\text { y } 39 \text { años. }\end{array}$ & & & + & + & \\
\hline $\begin{array}{l}\text { Lindland, } \\
\text { Nygaard, Bo, } \\
\text { (2007) }\end{array}$ & 12 & $\begin{array}{l}\text { Ejercicio de alta } \\
\text { intensidad de } \\
\text { edades promedio } \\
\text { de } 24 \text { años. }\end{array}$ & & & + & & \\
\hline $\begin{array}{l}\text { Luginbuehl et } \\
\text { al., (ㅁ16) }\end{array}$ & 10 & $\begin{array}{l}\text { Atletas de alto } \\
\text { impacto }\end{array}$ & & & + & & \\
\hline $\begin{array}{l}\text { Santos, } \\
\text { Caetano, }\end{array}$ & 58 & $\begin{array}{c}\text { Estudiantes de } \\
\text { educación física de } \\
\text { edad primerio de } \\
21,4 \text { años. }\end{array}$ & + & + & & & \\
\hline
\end{tabular}


Tavares,

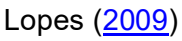

Fuente: Elaboración propia, 2017.

En el caso de los FR asociados al deporte, su práctica ha sido importante de analizar, ya que se observa que las características propias del mismo demostraron tener relación importante con la IUE.

Discusión

Debido a la incongruencia entre los países con mayor cantidad de publicaciones y el idioma predominante, se deduce que los investigadores prefieren publicar en el idioma inglés para tener mayor relevancia en el ámbito científico y ampliar su posibilidad de ser leídos.

A partir de los resultados obtenidos en la prevalencia de IUE en la población estudiada (entre 8 y $80 \%$ según los artículos recolectados por las investigadoras), se dificulta definir un valor específico, ya que se cuenta con diferentes tipos de metodologías, poblaciones estudiadas, y diversas definiciones del concepto de IUE. Dos de los artículos diferencian la prevalencia de IUE al realizar actividad deportiva, o al someter el PP a un esfuerzo extra, como sucede al estornudar, toser o reírse. En ambos casos, la IUE es mayor en estos tres últimos escenarios, lo cual puede implicar que, durante el deporte, el PP se encuentra más activo, sin embargo, esto sigue siendo una hipótesis (Bo, 2004). Asimismo, la mayoría de los artículos analizados presentan situaciones de alta prevalencia de IUE en la población estudiada, dando indicios que la magnitud de la problemática es importante de valorar.

En cuanto a los FR personales, Elliasson et al., (2002) concluyeron que existe cierta asociación directamente proporcional entre la edad de las trampolinistas y el desarrollo de IUE. Sin embargo, la población analizada por los investigadores comprende 35 mujeres con una edad media de 15 años, la cual se considera una edad muy temprana, por lo que el riesgo se le atribuye a otro factor. Partiendo de esto, las atletas de mayor edad son también las de mayor experiencia en el trampolinismo por lo que son capaces de realizar saltos más altos y piruetas más avanzadas, sometiendo a su PP a fuerzas mayores.

Por otra parte, al analizar las características del ciclo menstrual, Da Roza et al. (2015b) encontraron que, de 22 trampolinistas de élite, 16 presentaron IU. De éstas atletas, todas habían presentado la menarca a una edad más avanzada en comparación con las mujeres continentes. De la misma manera concuerdan con mayor cantidad de años de practicar el deporte y volúmenes más elevados de entrenamiento, lo cual podría explicar su reacción estrogénica.

Asimismo, Eliasson, Edner et al (2008) demostraron que la cantidad de años de realizar trampolinismo después de la menarca aumenta el riesgo de IUE con una media de 3 años y con un OR de 1,8. Además, las mujeres que manifestaron presentar fugas de orina durante su periodo de trampolinismo, así como posterior a éste, habían practicado dicho deporte por mayor tiempo después de la menarca.

De la misma forma, Eliasson, Edner et al (2008), encontraron que 17 de 28 trampolinistas mencionaron que la cantidad de orina liberada varía de acuerdo a su ciclo menstrual, mas no se especifica en qué periodo se presentan en menor o mayor medida. El tracto urinario y los genitales femeninos son sensibles al efecto de los esteroides sexuales, lo cual sugiere que la presencia de fugas de orina es más propensa a darse en la fase lútea, por la liberación de progesterona, que ha demostrado efectos de relajación de 
los tejidos pélvicos (Cardozo y Kelleher, 1994). De este modo, es probable que la IU se presente posterior a la menarca ya que ésta inicia la producción de la progesterona.

Al evaluar mujeres de tres deportes de alto impacto y un grupo control, Da Silva Borin et al. ( $\underline{2013})$ hallaron menor presión perineal en las basquetbolistas que en las voleibolistas y a su vez, el grupo control poseía la mayor capacidad de presión por encima de todos los grupos.

Por su parte cuatro de los artículos analizaron la incapacidad de detener el flujo urinario durante la micción, y todos sus resultados coincidieron en que éste era importante de valorar. Eliasson et al. (2002) asegura que las trampolinistas con mayores cantidades de orina perdida por episodio también se les imposibilitaba esta acción. Asimismo, Da Roza et al. (2015a) demuestra que las mujeres que practican mayores volúmenes de actividad física por semana presentan la misma incapacidad, en comparación con las sedentarias. Ésta característica puede estar ligada a disfunciones del PP, al tiempo de reacción del mismo y/o al debilitamiento de los músculos.

También, Eliasson, Elving et al (2008), mostraron una asociación evidente donde éste factor incrementa doblemente el riesgo de padecer incontinencia en mujeres nulíparas, mientras que en multíparas se incrementa cuatro veces. Por su parte, en su otra investigación concluye que las mujeres a las que se les dificulta detener el flujo urinario tienen el triple de probabilidad de padecer de IU (Eliasson, Edner et al. 2008).

En la investigación de Capson et al. (2011) se demuestra que la mayor activación tónica de la musculatura del piso pélvico se da en posición bípeda e hipolordótica, dando resultados significativamente más altos que la postura neutra. Por su parte, la presión intravaginal muestra un aumento significativo en las personas con una posición lumbopelvica hipolordótica, seguido por la hiperlordótica y de último, la neutra. Se sugiere que esto puede deberse a que la posición de la vejiga varía con la postura de hipolordosis, generando un ángulo mayor, que favorece este incremento de presión.

Asimismo, Eliasson, Elving et al (2008) aseguran que las nulíparas con diagnóstico de lumbalgia, tienen $>3$ prevalencia de IUE que aquellas que están sanas. En su estudio, es más común en aquellas que aseguran tener lumbalgia recurrente.

Eliasson, Edner et al (2008) demostraron que el estreñimiento fue el factor que se vio mayormente asociado con IU, triplicando el riesgo de padecerla en presencia del mismo. Esto se explica por el mal funcionamiento del intestino al comprimir la vejiga dificultando la retención urinaria (Higa, Lopes y Dos Reis, 2008). Asimismo, Markwell (2001) concluye que cuando existe dificultad para defecar puede ser el resultado de la baja actividad tónica del PP y del descenso perineal.

Dos estudios aseguran que el asma duplica y hasta el triplica el riesgo de IU (Eliasson, Elfving, Nordgren y Mattsson, 2008; Eliasson, Edner et al. 2008). Esto se puede explicar debido a que la enfermedad se asocia a la tos crónica, generando repetidas hiperpresiones abdominales por la interrelación que existe entre el diafragma y el piso pélvico. Entonces, al toser, el diafragma desciende con fuerza enviando esta presión al PP y provocando, a largo plazo, el debilitamiento del mismo.

En el caso de los FR asociados al deporte, el estudio Da Roza et al. (2015b) mencionan que las trampolinistas presentaron los episodios de fugas de orina al momento de comenzar a hacer dicho deporte, y en el momento en que están realizando el deporte. Con lo cual, Carls (2007), Dos Santos, Caetano, Fernandes, y de Moraes Lopes (2009) y Da Roza et al. (2015c) coinciden. Eliasson et al. (2002) agrega que la sintomatología aparece después de aprox. 2,5 años de estar practicando la misma. 
Los deportes de alto impacto se relacionan con una mayor predisposición de IUE. En la investigación realizada por Simeone et al. $\underline{2010}$ utilizada como apoyo a esta revisión, se asegura que según el grado de impacto que genere el deporte en el cuerpo de la deportista, así se tendrá mayor influencia en la incidencia de IUE (Simeone et al. 2010).

No obstante, Da Roza et al. (2015a) y Santos et al. (2009), mencionan una relación entre la natación y la IUE, lo cual se puede deber a que en la natación se involucran aumentos de presión intraabdominales, de manera sostenida y repetitiva.

Da Silva et al. (2013) demostraron que la presión perineal de las basquetbolistas es inferior a la de las voleibolistas, y este último inferior a la de las sedentarias. Por otro lado, Capson et al. (2011) demostraron que la presión intravaginal aumenta considerablemente en tareas que involucren atajar un objeto, muy por encima que la que genera la maniobra de Valsalva o toser. De esta forma es importante mencionar que trampolinismo, significa un riesgo para la debilidad del PP. Fozzatti et al. (2012) también encontraron que las mujeres que asisten al gimnasio con regularidad tenían episodios de fugas de orina, principalmente al realizar maniobras como saltar, el step y al correr.

Al investigar sobre el volumen de entrenamiento, varios autores coinciden en que existe una fuerte relación directamente proporcional entre los años de entrenamiento, la frecuencia de los episodios y la cantidad de orina perdida en cada uno de ellos. Da Roza et al. (2015b) concluyen que las que realizaban el deporte de 2 a 3 horas, 5 a 6 veces por semana mostraban cantidades moderadas de pérdida de orina con más frecuencia. El estudio de Eliason et al. (2002) y de Vitton et al. (2011) concuerdan con estos resultados.

Por otro lado, la mayoría de los artículos coincidieron en que el ejercicio vigoroso, provoca debilidad del PP y desencadena episodios de IUE, ya que se observó que la fuerza de contracción voluntaria del PP disminuye hasta en un $20 \%$. Los resultados sugieren, que ante esos parámetros de entrenamiento se desarrolla fatiga muscular de corto plazo, lo cual se recupera en aproximadamente 60 minutos de reposo (Lindland, Nygaard y Bø, 2007). Sin embargo, de acuerdo con Luginbuehl et al., (2016), puede deberse a una mayor actividad EMG de la musculatura del PP al presentarse actividades de mayor vigorosidad, por una acción refleja de la musculatura al verse sometida a impactos constantes. Mientras que intensidad de leve a moderada se observa menor actividad de esta musculatura predisponiendo a que se den fugas de orina.

Asimismo, Da Roza et al. (2015b) establecen que hay una relación directamente proporcional entre los años de entrenamiento y la frecuencia de los episodios y cantidad de fugas de orina en las trampolinistas. Kruger et al. (2007) y Eliasson et al. (2002) concuerdan, con más de 5 años y más de 2,5 años respectivamente.

Además, las investigaciones sugieren que aquellas atletas que entrenan a nivel competitivo poseen mayor frecuencia de padecer IU, tienen mayor movilidad del cuello de la vejiga y les cuesta más detener el flujo de orina durante la micción, por encima de aquellas que realizan ejercicio de manera recreativa o son sedentarias. El estudio de Da Roza et al. (2015a), muestra que las mujeres que competían tenían 2,53 más posibilidades de padecer IU que las inactivas, o que realizan menos de 30 minutos de actividad física por semana.

Por otro lado, Elliasson et al. (2002) mencionan que las fugas se presentan principalmente al final del entrenamiento, al realizar un nuevo movimiento y al realizar 
maniobras más complejas. Lo anterior apoya el hallazgo de Lindland et al. (2007) mencionado anteriormente.

Tabla 6. Mecanismos fisiológicos relacionados a los FR personales y asociados al deporte encontrados en los artículos científicos.

\begin{tabular}{|c|c|}
\hline $\begin{array}{l}\text { Factores de riesgo asociados a } \\
\text { IUE }\end{array}$ & Mecanismo fisiológico desencadenante \\
\hline \multicolumn{2}{|l|}{ Factores de riesgo personales } \\
\hline $\begin{array}{l}\text { Características del ciclo } \\
\text { menstrual }\end{array}$ & $\begin{array}{l}\text { Según los resultados del estudio de Elliasson et al. (2002) la } \\
\text { cantidad de orina liberada en las mismas varían de acuerdo } \\
\text { con su ciclo. menstrual. El estudio de Cardozo y Kelleher } \\
\text { (1994), confirma esta relación ya que descubrieron que tanto } \\
\text { el tracto urinario como los genitales femeninos son sensibles } \\
\text { al efecto de los esteroides sexuales porque poseen } \\
\text { receptores sensibles a la presencia de estrógeno y } \\
\text { progesterona. Partiendo de esto, se sugiere que la presencia } \\
\text { de fugas de orina es más propensa a darse en la fase lútea } \\
\text { ya que es donde se libera la mayor cantidad de } \\
\text { progesterona, la cual ha demostrado efectos de relajación de } \\
\text { los tejidos pélvicos. Por lo que es probable que la IUE se } \\
\text { presente posterior a la menarca ya que ésta inicia la } \\
\text { producción de la progesterona. }\end{array}$ \\
\hline Morfología lumbar y lumbalgia & $\begin{array}{l}\text { Un cambio en la posición lumbopélvica influencia tanto la } \\
\text { capacidad de contractilidad del piso pélvico, así como la } \\
\text { presión intravaginal que se genera durante la realización } \\
\text { estática y dinámica de tareas. } \\
\text { La mayor activación tónica de la musculatura del piso pélvico } \\
\text { se da en posición bípeda e hipolordótica, dando resultados } \\
\text { significativamente más altos que la postura neutra. Por otro } \\
\text { lado, al hacer una contracción voluntaria máxima se nota una } \\
\text { activación mayor del piso pélvico en la posición neutra, } \\
\text { mientras que tanto en hiperlordosis como hipolordosis se } \\
\text { presencia una disminución importante del registro } \\
\text { electromiográfico (EMG) del piso pélvico, inclusive al toser y } \\
\text { al reproducir la maniobra de Valsalva. } \\
\text { Por su parte, la presión intravaginal muestra un aumento } \\
\text { significativo en las personas con una posición lumbopelvica } \\
\text { hiporlordótica. Se sugiere que esto puede deberse a que la } \\
\text { posición de la vejiga varía con la postura de hipolordosis, } \\
\text { generando un ángulo mayor, que favorece este incremento } \\
\text { de presión. (Capson et al., 2011) }\end{array}$ \\
\hline Asma & $\begin{array}{l}\text { Enfermedades crónicas como el asma se asocian a la tos } \\
\text { crónica, lo cual genera repetidas hiperpresiones } \\
\text { abdominales por la interrelación que existe entre el } \\
\text { diafragma y el piso pélvico, donde, al toser, el diafragma } \\
\text { desciende con fuerza, enviando fuerza de presión al piso } \\
\text { pélvico y provocando, a largo plazo, el debilitamiento de este } \\
\text { (Eliasson, Elving et al., 2008, Eliasson, Edner et al., 2008). }\end{array}$ \\
\hline \multicolumn{2}{|l|}{$\begin{array}{l}\text { Factores de riesgo asociados } \\
\text { al deporte }\end{array}$} \\
\hline Tipo de deporte & $\begin{array}{l}\text { Los estudios encontraron que los deportes de alto impacto } \\
\text { mostraban mayor prevalencia de IUE lo cual puede deberse } \\
\text { a las hiperpresiones intraabdominales sostenidas y de } \\
\text { manera repetitiva (Almeida et al., } \underline{2015} \text { ). Por su parte, otro }\end{array}$ \\
\hline
\end{tabular}




\begin{tabular}{|l|l|}
\hline del & $\begin{array}{l}\text { estudio demostró que la presión intravaginal aumenta } \\
\text { considerablemente en tareas que involucren atajar un } \\
\text { objeto, muy por encima que la que genera la maniobra de } \\
\text { Valsalva o toser (Capson et al., 2011). }\end{array}$ \\
\hline $\begin{array}{l}\text { Características } \\
\text { entrenamiento }\end{array}$ & $\begin{array}{l}\text { Kruger et al. (2007) asegura que las deportistas que } \\
\text { entrenan por al menos 5 años presentan cambios en la } \\
\text { biomecánica y morfología de su suelo pélvico, generando } \\
\text { más probabilidades de perder involuntariamente orina } \\
\text { durante el esfuerzo. También se observó una disminución } \\
\text { de la presión perineal en aquellas deportistas que jugaban } \\
\text { mayor cantidad de partidos por año, y que invertían en su } \\
\text { semana mayor cantidad de minutos en entrenamientos de } \\
\text { cancha o en sesiones de fortalecimiento } \\
\text { independientemente del deporte que practicaran (Da Silva } \\
\text { Borin et al., 2013). }\end{array}$ \\
\hline Nivel de entrenamiento & $\begin{array}{l}\text { Un estudio encontró que las mujeres que entrenan de } \\
\text { manera competitiva deportes de alto impacto con una } \\
\text { intensidad alta, muestran mayor movilidad del cuello de la } \\
\text { vejiga, así como descenso del órgano en general. (Kruger } \\
\text { et al., } 2007)\end{array}$ \\
\hline Periodo del entrenamiento & $\begin{array}{l}\text { Lindland Ree et al. (2007) demostraron que la fuerza de los } \\
\text { músculos del suelo pélvico disminuye hasta en un 20\% en } \\
\text { contracciones voluntarias después de actividad física } \\
\text { vigorosa, principalmente al finalizar el entrenamiento, lo que } \\
\text { desarrolla fatiga muscular en éstos. }\end{array}$ \\
\hline
\end{tabular}

Fuente: Elaboración propia, 2019

\section{Conclusiones generales}

La prevalencia de IUE encontrada fue muy variada ya que se presentaron casos en los que solo el $9 \%$ de mujeres evaluadas la padecían, mientras que en otros la totalidad de la población la presentaba. Partiendo de esto, se dificulta definir la magnitud de la problemática, ya que se abarcan estudios con diferentes tipos de metodologías, poblaciones estudiadas y diferentes definiciones de incontinencia. Para futuras investigaciones se recomienda establecer una definición estandarizada de IUE para aumentar la validez de la investigación.

Se pudo observar que los resultados más significativos fueron encontrados mayormente en los factores deportivos o extrínsecos a la persona. Con respecto a los personales, se concluye que el estreñimiento y la lumbalgia comprendían los principales FR para padecer IUE, triplicando las posibilidades, seguidos por la inhabilidad de detener el flujo urinario. Igualmente, aquellas que practicaron deporte transcurridos más de tres años después de la menarca presentaron un riesgo de 1.8 veces mayor de padecer IUE.

Además, se concluye que el practicar deporte significa un riesgo relacionado a este padecimiento. Los deportes que presentaron mayor predisposición a padecer IUE, son los considerados de alto impacto, así como los que involucran atajar un objeto ya que aumentan considerablemente la presión intravaginal, aún por encima de la maniobra de Valsalva. 
En cuanto a las características del entrenamiento, la cantidad de años de practicar el deporte, y el volumen semanal de entrenamiento, mostraron mayor relevancia y a la vez son directamente proporcionales a la severidad del mismo. Asimismo, las deportistas que entrenaban a nivel competitivo reportaban episodios más frecuentes y mayor cantidad de orina perdida por cada uno de ellos.

Aún no existe suficiente evidencia acerca del funcionamiento del PP y la IUE en mujeres deportistas nulíparas, ni de la relación con el deporte. Sin embargo, los hallazgos de esta investigación refuerzan los estudios que ya se han realizado en los cuales se considera que el deporte de alto impacto tiene un papel importante como factor de riesgo para la IUE. Por ende, dados los resultados de la revisión sistemática y los hallazgos en cuanto a los mecanismos fisiológicos relacionados con el padecimiento, las autoras definen la IUE como la perdida de orina involuntaria ante un aumento de la presión intraabdominal sumado a características morfológicas y mecánicas del suelo pélvico que predisponen este fenómeno.

\section{Bibliografía}

(*) Estudios tomados en cuenta para la revisión

Almeida, M., Barra, A., Saltiel, F., Silva-Filho, A., Fonseca, A., y Figueiredo, E. (2015). Urinary incontinence and other pelvic floor dysfunctions in female athletes in Brazil: A cross-sectional study. Scandinavian Journal of Medicine Science in Sports, 26(9),1-8. doi: https://doi.org/10.1111/sms.12546

*Bo, K. (2004). Urinary Incontinence, Pélvic Floor Dysfunction, Exercise and Sport. Sports Medicine, 34(7), 451-464. doi: https://doi.org/10.2165/00007256-200434070-00004

Cabrera, M. (2006). La incontinencia urinaria en la mujer deportista de élite. Revista Iberoamericana de Fisioterapia y Kinesiología, 9(2), 78-89. doi: https://doi.org/10.1016/S1138-6045(06)73119-7

${ }^{*}$ Capson, A. C., Nashed, J., y Mclean, L. (2011). The role of lumbopelvic posture in pelvic floor muscle activation in continent women. Journal of Electromyography and Kinesiology, 21(1), 166-177. doi: https://doi.org/10.1016/j.jelekin.2010.07.017

Cardozo, L., y Kelleher, C. (1994). Sex hormones and the female lower urinary tract. Physiotherapy, 80(3), 135-138. doi: https://doi.org/10.1016/S0031-9406(10)61261-8

${ }^{*}$ Carls, C. (2007). The prevalence of stress urinary incontinence in high school and college age female athletes in the Midwest: implications for education and prevention. UROLOGIC NURSING, 27(1), 21-24. Recuperado de: https://www.suna.org/sites/default/files/download/members/unjarticles/2007/07feb/21 .pdf

*Da Roza, T., Brandão, S., Mascarenhas, T., Jorge, R. N., y Duarte, J. A. (2015a). Urinary incontinence and levels of regular physical exercise in young women. International journal of sports medicine, 36(09), 776-780. doi: https://doi.org/10.1055/s-00341398625 
*Da Roza, T., Brandão, S., Mascarenhas, T., Jorge, R. N., y Duarte, J. A. (2015b). Volume of training and the ranking level are associated with the leakage of urine in young female trampolinists. Clinical Journal of Sport Medicine, 25(3), 270-275. doi: https://doi.org/10.1097/JSM.0000000000000129

*Da Roza, T., Brandão, S., Oliveira, D., Mascarenhas, T., Parente, M., Duarte, J. A., y Jorge, R. N. (2015c). Football practice and urinary incontinence: relation between morphology, function and biomechanics. Journal of biomechanics, 48(9), 1587-1592. doi:https://doi.org/10.1016/j.jbiomech.2015.03.013

*Da Roza, T., Natal Jorge, R., Mascarenhas, T., \& Alberto Duarte, J. (2013). Urinary incontinence in sport women: from risk factors to treatment-a review. Current Women's Health Reviews, 9(2), 77-84.

*da Silva Borin, L. C. M., Nunes, F. R., y de Oliveira Guirro, E. C. (2013). Assessment of pelvic floor muscle pressure in female athletes. Pm\&r, 5(3), 189-193. doi: http://dx.doi.org/10.1016/j.pmrj.2012.09.001

${ }^{*}$ Dockter, M., Kolstad, A. M., Martin, K. A., y Schiwal, L. J. (2007). Prevalence of Urinary Incontinence: A Comparative Study of Collegiate Female Athletes and Non-Athletic Controls. Journal of Women's Health Physical Therapy, 31(1), 12-17. doi: https://doi.org/10.1097/01274882-200731010-00003

*Dos Santos, E. S., Caetano, A. S., Fernandes, M. D. C. G. C., y de Moraes Lopes, M. H. B. (2009). Incontinência urinária entre estudantes de educação física. Revista da Escola de Enfermagem da USP, 43(2), 307-312. doi: https://doi.org/10.1590/S008062342009000200008

${ }^{*}$ Eliasson, K., Edner, A., y Mattsson, E. (2008). Urinary incontinence in very young and mostly nulliparous women with a history of regular organised high-impact trampoline training: occurrence and risk factors. International Urogynecology Journal, 19(5), 687696. doi: https://doi.org/10.1007/s00192-007-0508-4

${ }^{*}$ Eliasson, K., Elfving, B., Nordgren, B., y Mattsson, E. (2008). Urinary incontinence in women with low back pain. Manual therapy, 13(3), 206-212. doi: https://doi.org/10.1016/j.math.2006.12.006

*Eliasson, K., Larsson, T., y Matsson, E. (2002). Prevalence of stress incontinence in nulliparous elite trampolinists. Scandinavian journal of Medicine \& Science in sports, 12(2), 106-110. doi: https://doi.org/10.1034/j.1600-0838.2002.120207.x

Elleuch, M., Ghattassi, I., Guermazi, M., Lahiani, J., Kassis, M., Dammak, J., y López, S. (1998). Stress incontinence in nulliparous sportswomen: an epidemiological inquirí concerning. Annales de readaptation et de medecine physique, 41(8), 479-484. Recuperado de: https://www.infona.pl/resource/bwmeta1.element.elsevier4d18079a-2f6d-35d3-bc97-059cf0bc9bfd

*Fozzatti, C., Ricceto, C., Hermann, V., Brancalion, M., Raimondi, M., Nascif, C., Marques, L., Palma, P. (2012). Prevalence study of stress urinary incontinence in women who perform high-impact exercises. International Urogynecology Journal, 23(12), 16871691. doi: http://dx.doi.org/10.1007/s00192-012-1786-z 
*Greydanus, D. E., Omar, H., \& Pratt, H. D. (2010). The adolescent female athlete: current concepts and conundrums. Pediatric Clinics, 57(3), 697-718. Doi: https://doi.org/10.1016/j.pcl.2010.02.005

*Heath, A., Folan, S., Ripa, B., Varriale, C., Bowers, A., Gwyer, J., \& Figuers, C. (2014). Stress urinary incontinence in female athletes. Journal of Women's Health Physical Therapy, 38(3), 104-109. Doi: 10.1097/JWH.0000000000000016

*Higa, R., Baena de Moraes Lopes, M. H., y dos Reis, M. J. (2008). Fatores de risco para incontinência urinária na mulher. Revista da Escola de Enfermagem da USP, 42(1). doi: http://dx.doi.org/10.1590/S0080-62342008000100025.

*Kruger, J. A., Dietz, H. P., y Murphy, B. A. (2007). Pelvic floor function in elite nulliparous athletes. Ultrasound in obstetrics \& gynecology, 30(1), 81-85. doi: http://dx.doi.org/ 10.1002/uog.4027

*Lindland Ree, M, Nygaard, I. y Bo, K. (2007) Muscular fatigue in the pelvic floor muscles after strenuous physical activity. Acta Obstet Gynecol Scand, 86(7),870-6. doi: http://dx.doi.org/10.1080/00016340701417281

*Luginbuehl, H., Naeff, R., Zahnd, A., Baeyens, J. P., Kuhn, A., y Radlinger, L. (2016). Pelvic floor muscle electromyography during different running speeds: an exploratory and reliability study. Archives of gynecology and obstetrics, 293(1), 117-124. doi: http://dx.doi.org/10.1007/s00404-015-3816-9

Markwell, S. J. (2001). Physical therapy management of pelvi/perineal and perianal pain syndromes. World journal of urology, 19(3), 194-199. doi: https://doi.org/10.1007/PL00007097

Martínez, S. (2012). Deporte femenino y suelo pélvico. Recuperado de https://ruc.udc.es/dspace/bitstream/handle/2183/9083/ActasGEXAFD10.pdf?sequen $\mathrm{ce}=1$ \&isAllowed $=\mathrm{y}$

Napal, C., Lara, C., Echeandía, B., Egaña, C., Lezaún, R., Sancho, B., y Recalde, A. (2004). Pasado, presente y futuro de la incontinencia urinaria. ENFURO, 89, 8-10. Recuperado de https://dialnet.unirioja.es/servlet/articulo?codigo=3100241

Nygaard, I., Thompson, F., Svengalis, S., y Albright, S. (1994). Urinary Incontinente in Elite Nulliparous Athletes. The American College of Obstetricians and Gynecologistics, 84(2), 183-187. Recuperado de https://www.ncbi.nlm.nih.gov/pubmed/8041527

Rial, T., y Riera, T. (2012). Prevalencia y abordaje desde el ejercicio físico de la incontinencia urinaria en mujeres deportistas. EFDeportes.Com, 164, 1-10. Recuperado de https://www.efdeportes.com/efd164/incontinencia-urinaria-enmujeres-deportistas.htm

Santos, E. S. D., Caetano, A. S., Tavares, M. D. C. G. C., y Lopes, M. H. B. D. M. (2009). Urinary incontinence among physical education students. Revista da Escola de Enfermagem da USP, 43(2), 307-312. doi: http://dx.doi.org/10.1590/S008062342009000200008 
Simeone, C., Moroni, A., Pettenò, A., Antonelli, A., Zani, D., Orizio, C., y Cosciani Cunico, S. (2010). Occurrence rates and predictors of lower urinary tract symptoms and incontinence in female athletes. Urología, 77(2), 139-146. doi: https://doi.org/10.1177/039156031007700210

Vitton, V., Baumstarck-Barrau, K., Brardjanian, S., Caballe, I., Bouvier, M., y Grimaud, J. (2011). Impact of high-level sport practice on anal incontinence in a healthy young female population. Journal of Women's Health, 5(20), 757-763. doi: https://doi.org/10.1089/jwh.2010.2454 